\title{
A Pulsewidth Modulation Based Integral Sliding Mode Current Controller for Boost Converters
}

\author{
Siew-Chong Tan, Y. M. Lai, Chi K. Tse, and Chi Kin Wu \\ Department of Electronic and Information Engineering \\ The Hong Kong Polytechnic University, Hong Kong, China \\ email: ensctan@eie.polyu.edu.hk
}

\begin{abstract}
This paper proposes a fixed-frequency pulsewidth-modulation based sliding mode current controller for boost converters. The aim of having such a nonlinear controller is to support the control applications of boost converters requiring a fast dynamical response over a wide range of operating conditions. The methods of modeling the system and translation of the sliding mode control equations for pulsewidth modulation implementation are illustrated. It is shown that the proposed controller is easily realized with simple analog circuitries. Experimental results are presented to validate the theoretical design and to illustrate the strength of the proposed controller in wide operating conditions.
\end{abstract}

\section{INTRODUCTION}

Boost-type converters operating in the continuous conduction mode (CCM) inherit the right-half-plane-zero (RHPZ) characteristic in their duty-cycle-to-output-voltage transfer functions [1]. This typically makes the dynamic response of the system sluggish, particularly when the mode of control is based solely on controlling the output voltage, i.e., voltage mode control [1]. This is because with the voltage mode control, the crossover frequency must be well above the resonant frequency to stabilize the system [2]. This complicates the design and limits the range of applicable compensation network, considering that the crossover frequency is concurrently restricted by the presence of the RHPZ [2]. Hence, in voltage controlled RHPZ converters, designers are typically forced to choose low gain bandwidth (slow response) types of compensation network for their controllers. These properties are true to both linear and nonlinear types of voltage controllers.

A solution to achieving fast dynamical response in RHPZ converter systems is to employ the current mode control [3]. Since it is not necessary for the current mode control to have a crossover frequency above the filter resonant frequency, simpler compensation and a higher gain bandwidth over a comparable voltage mode circuit can be achieved [2], [3]. Consequently, current mode control allows the possible attainment of a faster dynamic responding RHPZ system for a given switching frequency than the voltage mode control [3]. Additionally, the adoption of the inner current loop also introduces a nonlinear state feedback term which makes the current mode control semi-nonlinear, since the voltage loop is maintained linear. This is unlike conventional voltage mode control which is entirely linear. Hence, current mode control supports converter applications over a wider range of operating condition than linear voltage mode control. However, it still does not fully support converter applications over a very wide range of operating conditions. This spurs numerous investigations into various nonlinear controllers for power converters.

Among them, the sliding mode (SM) controller is deemed to be a better candidate than the others for its relative ease of implementation, as well as its excellent robust and stability properties [4]-[6]. In particular, the fixed-frequency pulsewidth modulation (PWM) based SM controllers are more suited for practical implementation in power converters [4], [7]-[12]. The main operating mechanism of such controllers is a pulsewidth modulator that employs a control signal derived from SM control technique. Originated from the ideas of various papers [4], [7], [8], [13], [14], an improved form of this controller has been recently experimentally demonstrated on the buck [11] and the boost [12] converters using the SM voltage control configuration. Even though, the experimental results show that the so-called PWM based SM voltage controller offers good large-signal control performances in both buck and boost converters, it is easily understood that the application of this controller to boost converters is nonoptimal. It is necessary to take into consideration the current mode control variables when formulating the PWM based SM controllers for converters with RHPZ, so that optimal performance in such system can be obtained.

In view of this, a fixed-frequency PWM based SM current controller is proposed in this paper to supplement the existing class of PWM based SM voltage controllers for controlling DC-DC converters with RHPZ. The main objective of having such a nonlinear controller is to provide the control support for RHPZ converters which have 1) wide operating conditions that cannot be satisfactorily controlled by conventional PWM current mode controller, and 2) fast response requirement that cannot be met by the PWM based SM voltage controller.

\section{Proposed Sliding Mode Current Controller}

The proposed SM current controller employs both the output voltage error and the inductor current error as the controlled state variables. The adoption of the output voltage error as the state variable allows the output voltage to be accurately regulated, whereas the inductor current error allows the inductor current to be maintained closely to the desired reference inductor current. Similar to the conventional current mode control, the reference inductor current profile in the proposed controller is generated using the amplified output voltage error. This section proceeds to cover the theoretical derivation of the PWM based SM current controller from its conventional hysteresis modulation (HM) form. The derivation of the existence and stability conditions are also illustrated. 


\section{A. In Conventional Hysteresis Modulation Form}

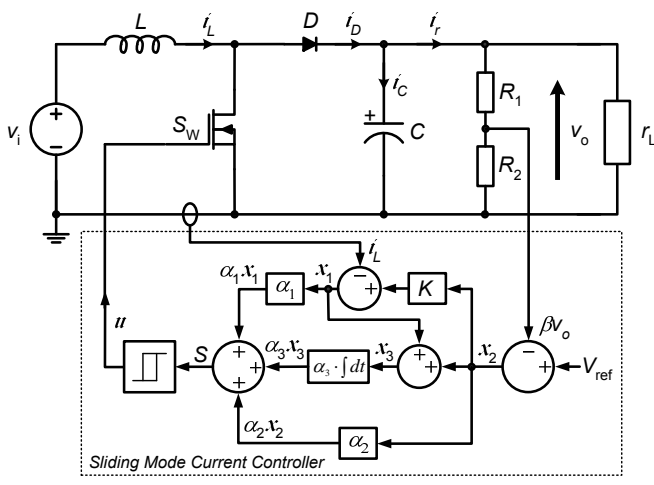

(a) In hysteresis modulation form

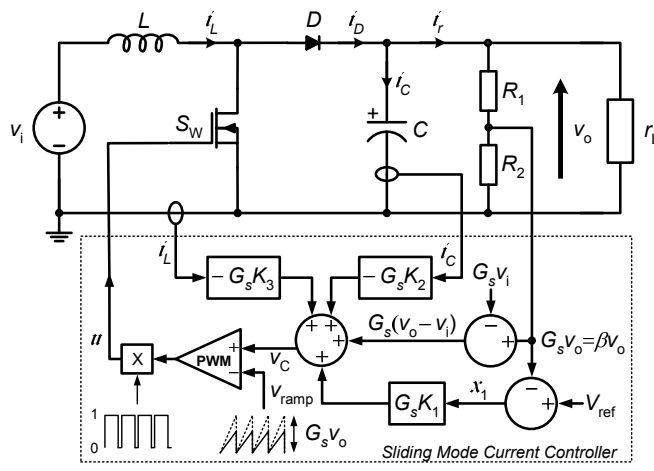

(b) In pulsewidth modulation form

Fig. 1. Proposed sliding mode current controller for boost converters.

Fig. 1(a) shows a schematic diagram of the proposed SM current controller for boost converters in its conventional HM form. The proposed controller adopts a control law

$$
u= \begin{cases}1=\text { 'ON' } & \text { when } S>0 \\ 0={ }^{\prime O F F}, & \text { when } S<0\end{cases}
$$

where $u$ is the state of power switch $S_{\mathrm{W}}$, and $S$ is the instantaneous state variable's trajectory expressed as

$$
S=\alpha_{1} x_{1}+\alpha_{2} x_{2}+\alpha_{3} x_{3}
$$

where $\alpha_{1}, \alpha_{2}$, and $\alpha_{3}$ represents the desired control parameters termed as sliding coefficients. Here, the adopted controlled state variables are the current error $x_{1}$, the voltage error $x_{2}$, and the integral of the current and the voltage errors $x_{3}$. They are expressed as

$$
\left\{\begin{array}{l}
x_{1}=i_{\text {ref }}-i_{L}=K\left[V_{\text {ref }}-\beta v_{\mathrm{o}}\right]-i_{L} \\
x_{2}=V_{\text {ref }}-\beta v_{\mathrm{o}} \\
x_{3}=\int\left[x_{1}+x_{2}\right] d t
\end{array}\right.
$$

where $V_{\text {ref }}$ and $v_{\mathrm{o}}$ denote the reference and instantaneous output voltages respectively; $\beta$ denotes the feedback network ratio; and $i_{\text {ref }}$ and $i_{L}$ denote the instantaneous reference and inductor currents respectively, where $i_{\text {ref }}=K\left[V_{\text {ref }}-\beta v_{\mathrm{o}}\right]$ and $K$ is the amplified gain of the voltage error. It should be mentioned that the use of integral control here is optional. As in conventional current mode control, a sufficiently large value of $K$ in the outer voltage loop is sufficient to ensure an excellent regulation of the output voltage with a negligible steady-state error.

Ideally, at an infinitely high switching frequency, only the controlled variables $x_{1}$ and $x_{2}$ are required in the SM current controller to ensure that both the output voltage and inductor current are regulated to follow exactly their references, i.e., $v_{\mathrm{o}}=V_{\text {ref }}$ and $i_{L}=i_{\text {ref. However, in the case of finite- }}$ frequency or fixed-frequency SM controllers, the control is imperfect. Steady-state errors exist in both the output voltage error and the inductor current error such that $v_{\mathrm{o}} \neq V_{\text {ref }}$ and $i_{L} \neq i_{\text {ref }}$. Therefore, an integral term of these errors $x_{3}$ has been introduced into the SM current controller as an additional controlled state variable to reduce these steady-state errors. This is commonly known as integral SM control [5].

Next, the substitution of the boost converter's behavioral models under CCM into the time differentiation of (3) gives

$$
\left\{\begin{array}{l}
\dot{x_{1}}=\frac{d\left[i_{\mathrm{ref}}-i_{L}\right]}{d t}=-\frac{\beta K}{C} i_{C}-\frac{v_{\mathrm{i}}-\bar{u} v_{\mathrm{o}}}{L} \\
\dot{x_{2}}=\frac{d\left[V_{\mathrm{ref}}-\beta v_{\mathrm{o}}\right]}{d t}=-\frac{\beta}{C} i_{C} \\
\dot{x_{3}}=x_{1}+x_{2}=(K+1)\left[V_{\mathrm{ref}}-\beta v_{\mathrm{o}}\right]-i_{L}
\end{array}\right.
$$

where $\bar{u}=1-u$ is the inverse logic of $u$; $v_{\mathrm{i}}$ denotes the instantaneous input voltage; $i_{C}$ denotes the instantaneous capacitor current; and $C$ and $L$ denote the capacitance and inductance of the converters respectively. Also, with respect to the converter, $r_{\mathrm{L}}$ denotes the instantaneous load resistance and $i_{r}$ denotes the instantaneous load currents.

\section{B. In Proposed Pulse-Width Modulation Form}

Conventional implementation of the SM current controller in HM form requires only control equations (1) and (2). For implementation in PWM form, however, an indirect translation of the SM control law is required. The detailed description of this translation technique is provided in [11], [12]. Following the same approach, the equivalent control signal of the proposed SM current controller can be obtained by equating the time differentiation of (2) to zero, i.e., $\dot{S}=0$ to give

$$
\begin{array}{r}
u_{\text {eq }}=1-\frac{\beta L}{C v_{\mathrm{o}}}\left(K+\frac{\alpha_{2}}{\alpha_{1}}\right) i_{C}-\frac{v_{\mathrm{i}}}{v_{\mathrm{o}}}+\frac{\alpha_{3}}{\alpha_{1}} \frac{L}{v_{\mathrm{o}}}(K+1) \times \\
{\left[V_{\mathrm{ref}}-\beta v_{\mathrm{o}}\right]-\frac{\alpha_{3}}{\alpha_{1}} \frac{L}{v_{\mathrm{o}}} i_{L}}
\end{array}
$$

where $u_{\text {eq }}$ is continuous and bounded by 0 and 1 . Substitution of (5) into $0<u_{\mathrm{eq}}<1$ and multiplying by $v_{\mathrm{o}}$ gives

$$
\begin{aligned}
0<u_{\mathrm{eq}}{ }^{*}= & -\frac{\beta L}{C}\left(K+\frac{\alpha_{2}}{\alpha_{1}}\right) i_{C}+\frac{\alpha_{3}}{\alpha_{1}} L(K+1) \times \\
& {\left[V_{\text {ref }}-\beta v_{\mathrm{o}}\right]-\frac{\alpha_{3}}{\alpha_{1}} L i_{L}+\left[v_{\mathrm{o}}-v_{\mathrm{i}}\right]<v_{\mathrm{o}} }
\end{aligned}
$$

Finally, the mapping of the equivalent control function (6) onto the duty ratio control $d$, where $0<d=\frac{v_{\mathrm{c}}}{\hat{v}_{\text {ramp }}}<1$, gives the following relationships for the control signal $v_{\mathrm{c}}$ and ramp signal $\hat{v}_{\text {ramp }}$ for the implementation of the SM current controller in PWM form:

$$
\left\{\begin{array}{l}
v_{\mathrm{c}}=-\frac{\beta L}{C}\left(K+\frac{\alpha_{2}}{\alpha_{1}}\right) i_{C}+\frac{\alpha_{3}}{\alpha_{1}} L(K+1) \times \\
\quad\left[V_{\text {ref }}-\beta v_{\mathrm{o}}\right]-\frac{\alpha_{3}}{\alpha_{1}} L i_{L}+\left[v_{\mathrm{o}}-v_{\mathrm{i}}\right] \\
\hat{v}_{\text {ramp }}=v_{\mathrm{o}}
\end{array} .\right.
$$


A close inspection of these equations reveals that they are unsuitable for direct circuit implementation, especially when the input and the output voltages are high. Hence, in terms of practical implementation, it is necessary to scale down the equation to a level that conforms to the chip level voltage standard. Using a factor of $0<G_{s}<1$, (7) can be rewritten as

$$
\left\{\begin{array}{l}
v_{\mathrm{c}}=G_{s} K_{1}\left[V_{\mathrm{ref}}-\beta v_{\mathrm{o}}\right]-G_{s} K_{2} i_{C} \\
\quad-G_{s} K_{3} i_{L}+G_{s}\left[v_{\mathrm{o}}-v_{\mathrm{i}}\right] \\
\hat{v}_{\text {ramp }}=G_{s} v_{\mathrm{o}}
\end{array}\right.
$$

where $K_{1}=\frac{\alpha_{3}}{\alpha_{1}} L(K+1) ; K_{2}=\frac{\beta L}{C}\left(K+\frac{\alpha_{2}}{\alpha_{1}}\right)$; and $K_{3}=\frac{\alpha_{3}}{\alpha_{1}} L$ are the fixed gain parameters in the proposed controller. Fig. 1(b) shows the derived PWM based SM current controller for the boost converters. The design of the controller is based on equation (8) and the assumption $\beta=G_{s}$. A noteworthy comment is that two current sensors are required in its implementation. This is the main drawback of using this controller as compared to the PWM based SM voltage controller, which requires only the sensing of the capacitor current. Yet, the additional current sensing required on the inductor current is actually the main component constituting to a faster responding RHPZ converter system.

\section{Existence Condition}

The hitting condition has been satisfied by the control law in (1). It is necessary to derive the existence condition, which determines the ranges of employable sliding coefficients for the SM current controller before the design of the gain parameters $K_{1}, K_{2}$, and $K_{3}$ can be proceeded. To ensure the existence of SM operation, the local reachability condition $\lim _{S \rightarrow 0} S \cdot \dot{S}<0$ must be satisfied. The substitution of the time differentiation of (2) into this condition gives

$$
\left\{\begin{array}{c}
\alpha_{1}\left[-\frac{\beta K}{C} i_{C}-\frac{v_{\mathrm{i}}}{L}\right]-\alpha_{2} \frac{\beta}{C} i_{C} \\
+\alpha_{3}\left[(K+1)\left[V_{\mathrm{ref}}-\beta v_{\mathrm{o}}\right]-i_{L}\right]<0 \\
\alpha_{1}\left[-\frac{\beta K}{C} i_{C}-\frac{v_{\mathrm{i}}-v_{\mathrm{o}}}{L}\right]-\alpha_{2} \frac{\beta}{C} i_{C} \\
+\alpha_{3}\left[(K+1)\left[V_{\mathrm{ref}}-\beta v_{\mathrm{o}}\right]-i_{L}\right]>0
\end{array}\right.
$$

which can be simplified as

$$
0<v_{\mathrm{i}}-K_{1}\left[V_{\text {ref }}-\beta v_{\mathrm{o}}\right]+K_{2} i_{C}-K_{3} i_{L}<v_{\mathrm{o}} .
$$

The selection of the control gains of the controller must comply the inequality. In the case of designing an SM controller with a static sliding surface, a practical approach is to design the sliding coefficients to meet the existence conditions for steady-state operations [12], [15], [16]. Under such a consideration, the time varying state variables $i_{C}, i_{L}$, $v_{\mathrm{i}}$, and $v_{\mathrm{O}}$ can be respectively substituted with their expected maximum/minimum or steady-state parameters, which can be derived from the design specification. This gives

$$
\left\{\begin{array}{c}
0<v_{\mathrm{i}(\min )}-K_{1}\left[V_{\mathrm{ref}}-\beta v_{\mathrm{o}(\mathrm{SS})}\right] \\
+K_{2} i_{C(\min )}-K_{3} i_{L(\max )} \\
v_{\mathrm{i}(\max )}-K_{1}\left[V_{\mathrm{ref}}-\beta v_{\mathrm{o}(\mathrm{SS})}\right] \\
+K_{2} i_{C(\max )}-K_{3} i_{L(\min )}<v_{\mathrm{o}(\mathrm{SS})}
\end{array}\right.
$$

where $v_{\mathrm{i}(\max )}$ and $v_{\mathrm{i}(\min )}$ denotes the maximum and minimum input voltages respectively; $v_{\mathrm{o}(\mathrm{SS})}$ denotes the expected steadystate output voltage which is basically a DC parameter of a small error from the desired reference voltage $V_{\text {ref }}$; and $i_{L(\max )}, i_{L(\min )}, i_{C(\max )}$, and $i_{C(\min )}$ are respectively the maximum and minimum inductor and capacitor currents when the converter is operating at full-load condition. The compliance of the inequalities in (11) assures the existence of the SM operation to occur at least in the small region of the origin for all operating conditions up to full load.

\section{Stability Condition}

Unlike in SM voltage controllers where the selection of the sliding coefficients (control gains) to satisfy the stability condition is automatically performed by designing the system for some desired dynamic properties [11], [12], [17], the same approach cannot be adopted for designing the SM current controllers. This is because the motion equation (derived from $S=0$ ) of the SM current controllers, which composes both the current and voltage state variables, is highly nonlinear and cannot be easily solved through analytical method. A different approach based on the equivalent control method is adopted [5]. This is to firstly derive for the ideal sliding dynamics of the system, and then doing an analysis on its equilibrium point, which finally allows the stability condition to be obtained [16].

1) Ideal Sliding Dynamics: The replacement of $\bar{u}$ by $\bar{u}_{\mathrm{eq}}$ (so-called equivalent control method) into the original boost converter's description under CCM operation converts the discontinuous system into an ideal SM continuous system:

$$
\left\{\begin{array}{l}
L \frac{d i_{L}}{d t}=v_{\mathrm{i}}-\bar{u}_{\mathrm{eq}} v_{\mathrm{o}} \\
C \frac{d v_{\mathrm{o}}}{d t}=i_{L} \bar{u}_{\mathrm{eq}}-\frac{v_{\mathrm{o}}}{r_{\mathrm{L}}}
\end{array} .\right.
$$

Then, the substitution of (5) into (12) gives

$$
\left\{\begin{array}{l}
L \frac{d i_{L}}{d t}=K_{1}\left[V_{\text {ref }}-\beta v_{\mathrm{o}}\right]-K_{2} i_{C}-K_{3} i_{L} \\
C \frac{d v_{\mathrm{o}}}{d t}=\frac{i_{L}}{v_{\mathrm{o}}}\left[K_{2} i_{C}+v_{\mathrm{i}}-K_{1}\left[V_{\text {ref }}-\beta v_{\mathrm{o}}\right]+K_{3} i_{L}\right] \\
-\frac{v_{\mathrm{o}}}{r_{\mathrm{L}}}
\end{array}\right.
$$

which represents the ideal sliding dynamics of the SM current controlled boost converter.

2) Equilibrium Point Analysis: Assume that there exists a stable equilibrium point on the sliding surface on which the ideal sliding dynamics eventually settled. At this point of equilibrium, there will not be any change in the system's dynamics, i.e., $\frac{d i_{L}}{d t}=0$ and $\frac{d v_{\mathrm{o}}}{d t}=0$. Under such assumptions, the state equations in (13) can be equated to give

$$
\frac{K_{3}}{v_{\mathrm{i}} r_{\mathrm{L}}} v_{\mathrm{o}}^{2}+\beta K_{1} v_{\mathrm{o}}+\left(K_{2} i_{C}-K_{1} V_{\text {ref }}\right)=0 .
$$

Solving the quadratic equation for $v_{\mathrm{o}}$ leads to

$$
v_{\mathrm{o}}=\frac{-\beta K_{1} \pm \sqrt{\left(\beta K_{1}\right)^{2}-4\left(\frac{K_{3}}{v_{\mathrm{i}} r_{\mathrm{L}}}\right)\left(K_{2} i_{C}-K_{1} V_{\text {ref }}\right)}}{2\left(\frac{K_{3}}{v_{\mathrm{i}} r_{\mathrm{L}}}\right)} .
$$

The positive real solution of this equation is the stable equilibrium point of the output voltage. Hence, taking into 
consideration the actual specification of the converter, the following constraints can be inferred:

$$
\left\{\begin{array}{l}
K_{3}>0 \text { (non-singularity) } \\
\sqrt{\left(\beta K_{1}\right)^{2}-4\left(\frac{K_{3}}{v_{\mathrm{i}} r_{\mathrm{L}}}\right)\left(K_{2} i_{C}-K_{1} V_{\mathrm{ref}}\right)}>\beta K_{1} \\
\quad \Rightarrow \quad 0<K_{2}<\frac{V_{\mathrm{ref}}}{i_{C(\max )}} K_{1} \quad\left(v_{\mathrm{O}}\right. \text { must be positive) }
\end{array}\right.
$$

In essence, the existence (11) and the stability (16) conditions form the basis for the selection and design of the control gains of the proposed SM current controller in terms of the converter's specification. Specifically, the stability condition (16) provides the lower limits of the control gains while the existence condition (11) provides the upper limits.

\section{E. Selection of Sliding Coefficients $K_{1}, K_{2}$, and $K_{3}$}

Next, to relief the difficulty of designing the control gains based solely on the upper and lower limits, computer simulation and experiments were performed to study the effects of the various control gains on the response of the output voltage. It is observed that

a) an increment in $K_{1}$ improves the steady-state regulation but causes the transient response to be more oscillatory with a higher overshoot, thus prolonging the steady-state settling time (see Fig. 2(a));

b) an increment in $K_{2}$ improves the steady-state regulation and also makes the transient response less oscillatory with a lower overshoot, thus shortening the steady-state settling time (see Fig. 2(b)). However, the range of possible values for $K_{2}$ is very small (limited by the bidirectional capacitor current);

c) and a relatively small increment in $K_{3}$ can have a moderate reduction in the oscillation of the transient response and also significant shortening of the steadystate settling time. However, the steady-state regulation is deteriorated (see Fig. 2(c)).

Hence, in accordance to the observation, a heuristic but practical approach of designing the control gains is to firstly select the highest possible values of $K_{1}$ and $K_{2}$, and an arbitrary low value of $K_{3}$, without compromising the existence or stability condition for the full-load condition. Then, having the converter operated at the nominal input voltage and step load changes between the minimum and maximum loading condition, both the values of $K_{1}$ and $K_{3}$ can be up or down tuned to fit the converter's performance to the desired profile. As in conventional controllers, such tuning of the control parameters is typically required in initial prototyping stage to ensure that the converter responses in the desired manner before actual application or mass production. However, an advantage of the SM current controller over conventional controller is that once the parameters are decided for the nominal condition, the large-signal property of the SM current controller will ensure a relatively consistent dynamical behavior for all operating conditions, as in the SM voltage controller. This eliminates the need for re-tuning the parameters to compromise for other operating conditions, as required in conventional controllers.

\section{F. Additional Remarks}

The proposed SM current controller offers a fast dynamical response to converters with RHPZ by using the inductor current as a state variable in its control. This allows the controller to also inherit the other advantages of controlling current, namely, the over-current and audiosusceptibility protection properties. On the other hand, being a form of nonlinear controller, the SM current controller is capable of handling large-signal perturbations with excellent consistency in its dynamical responses. Hence, it is worthwhile to repeat that the targeted application of this controller is to provide the control support for RHPZ converters requiring a fast response over a wide range of operating conditions, which cannot be satisfactorily controlled by either the current mode controller or the PWM based SM voltage controller. This serves the main justification for its adoption in actual application, considering that it undesirably requires an additional current sensor and also requires a more complicated circuit architecture than the other two. Table I summarizes the general aspects of the performances and properties between the proposed controller and various PWM controllers.

\section{EXPERIMENTAL RESULTS AND DISCUSSIONS}

In this section, experimental results of the proposed PWM based SM current controller is provided to validate the theoretical design. Based on equation (8), the controller is constructed using simple analog ICs and operational amplifiers (see Fig. 1(b)) for a $100 \mathrm{~W}$ boost converters with specification shown in Table II. The control parameters adopted are $V_{\text {ref }}=6 \mathrm{~V}$, $G_{s}=\beta=\frac{1}{8}, K_{1}=80, K_{2}=3.12$, and $K_{3}=2.67$. They are chosen to comply with the design restrictions in (11) and (16), and have been fine tuned to respond to a desired regulation and dynamic response.

TABLE II

SPECIFICATION OF BOOST CONVERTER

\begin{tabular}{|lcc|}
\hline Description & Parameter & Nominal Value \\
\hline Input voltage & $v_{\mathrm{i}}$ & $24 \mathrm{~V}$ \\
Capacitance & $C$ & $230 \mu \mathrm{F}$ \\
Capacitor ESR & $c_{r}$ & $69 \mathrm{~m} \Omega$ \\
Inductance & $L$ & $300 \mu \mathrm{H}$ \\
Inductor resistance & $l_{r}$ & $0.14 \Omega$ \\
Switching frequency & $f_{\mathrm{S}}$ & $200 \mathrm{kHz}$ \\
Minimum load resistance (full load) & $r_{\mathrm{L}(\min )}$ & $24 \Omega$ \\
Maximum load resistance $(10 \%$ load) & $r_{\mathrm{L}(\max )}$ & $240 \Omega$ \\
Desired output voltage & $V_{\mathrm{od}}$ & $48 \mathrm{~V}$ \\
\hline
\end{tabular}

\section{A. Regulation Performance}

A tabulation of the data in terms of the load and line regulation properties is also given in Tables III and IV respectively. According to Table III, the maximum load-regulation error occurs at $v_{\mathrm{i}}=20 \mathrm{~V}$, with a deviation of $2.38 \%$ from $v_{\mathrm{o}(\mathrm{nom})}$. Similarly, it can be found from Table IV that the maximum line-regulation error occurs at minimum load $r_{\mathrm{L}}=240 \Omega$, with a deviation of $0.29 \%$ from $v_{\mathrm{o}(\mathrm{nom})}$.

\section{B. Dynamic Performance}

Figs. 3(a)-3(i) show the dynamic behavior of the PWM based SM current controlled boost converter at various input 


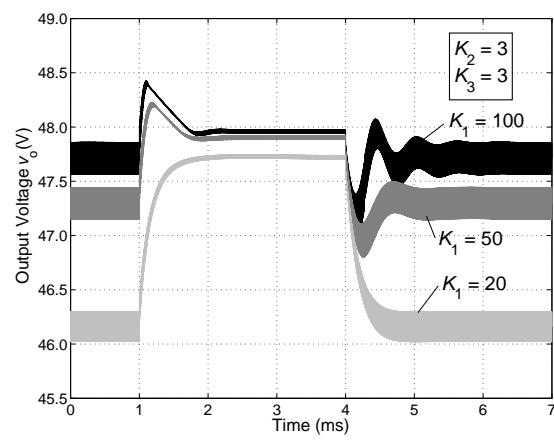

(a) Different $K_{1}$ settings

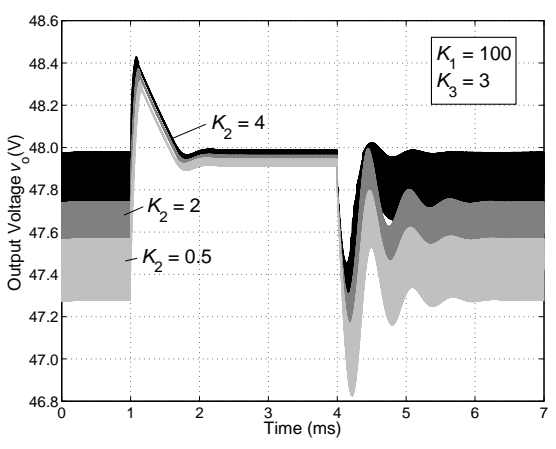

(b) Different $K_{2}$ settings

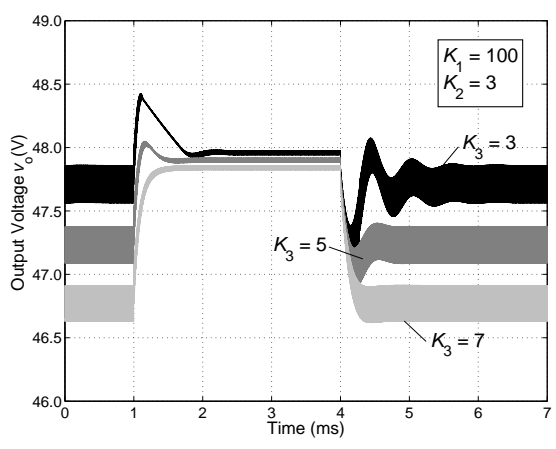

(c) Different $K_{3}$ settings

Fig. 2. Simulated waveforms of the output voltage $v_{\mathrm{o}}$ of a boost converter under the proposed SM current controller at various control gains settings.

TABLE I

A Comparison of The Various FiXed-Frequency PWM Control Schemes

\begin{tabular}{|c|c|c|c|c|}
\hline Category of Comparison & Voltage Mode & $\begin{array}{c}\text { Peak Current Mode/ } \\
\text { Average Current Mode }\end{array}$ & $\begin{array}{c}\text { Sliding Mode } \\
\text { Voltage Controller }\end{array}$ & $\begin{array}{c}\text { Sliding Mode } \\
\text { Current Controller }\end{array}$ \\
\hline type of control & linear & some nonlinearity & nonlinear & nonlinear \\
\hline compensation design & difficult & moderate/difficult & easy & moderate \\
\hline steady-state line and load regulation & average & good & average & average \\
\hline large-signal dynamic response consistency & poor & average & good & good \\
\hline gain bandwidth in RHPZ converter & low & relatively higher & low & one \\
\hline number of current sensing & nil & one & moderate & moderate \\
\hline noise-to-signal ratio & low & high/moderate & external circuit & inherent \\
\hline current protection & external circuit & inherent & existing & inherent \\
\hline audiosusceptibility protection & external scheme & inherent & &
\end{tabular}

TABLE III

LOAD REGULATION PROPERTY: OUTPUT VOLTAGE AT NOMINAL OPERATING CONDITION $v_{\mathrm{i}}=24 \mathrm{~V}$ AND $r_{\mathrm{L}}=24 \Omega$ IS $v_{\mathrm{o}(\mathrm{nom})}=47.45 \mathrm{~V}$.

\begin{tabular}{|c|c|c|}
\hline Input Voltage & Voltage Deviation: $\Delta v_{\mathrm{o}}=v_{\mathrm{o}(240 \Omega)}-v_{\mathrm{o}(24 \Omega)}$ & $\%$ Change: $\frac{\Delta v_{\mathrm{o}}}{v_{\mathrm{o}(\text { nom })}} \times 100 \%$ \\
\hline$v_{\mathrm{i}}=20 \mathrm{~V}$ & $1.13 \mathrm{~V}$ & $2.38 \%$ of $v_{\mathrm{o}(\mathrm{nom})}$ \\
\hline$v_{\mathrm{i}}=24 \mathrm{~V}$ & $0.82 \mathrm{~V}$ & $1.73 \%$ of $v_{\mathrm{o}(\mathrm{nom})}$ \\
\hline$v_{\mathrm{i}}=28 \mathrm{~V}$ & $0.35 \mathrm{~V}$ & $0.74 \%$ of $v_{\mathrm{o}(\mathrm{nom})}$ \\
\hline
\end{tabular}

TABLE IV

LINE REGULATION PROPERTY: OUTPUT VOLTAGE AT NOMINAL OPERATING CONDITION $v_{\mathrm{i}}=24 \mathrm{~V}$ AND $r_{\mathrm{L}}=24 \Omega$ IS $v_{\mathrm{o}(\mathrm{nom})}=47.45 \mathrm{~V}$.

\begin{tabular}{|c|c|c|}
\hline Loading Condition & Voltage Deviation: $\Delta v_{\mathrm{o}}=v_{\mathrm{o}\left(\mathrm{v}_{\mathrm{i}}=20 \mathrm{~V}\right)}-v_{\mathrm{o}\left(\mathrm{v}_{\mathrm{i}}=28 \mathrm{~V}\right)} \%$ Change: $\frac{\Delta v_{\mathrm{o}}}{v_{\mathrm{o}(\text { nom })}} \mathrm{x} 100 \%$ \\
\hline Minimum load $(240 \Omega)$ & $0.40 \mathrm{~V}$ & $0.84 \%$ of $v_{\mathrm{o}(\mathrm{nom})}$ \\
\hline Half load $(48 \Omega)$ & $0.27 \mathrm{~V}$ & $0.57 \%$ of $v_{\mathrm{o}(\mathrm{nom})}$ \\
\hline Full load $(24 \Omega)$ & $0.14 \mathrm{~V}$ & $0.29 \%$ of $v_{\mathrm{o}(\mathrm{nom})}$ \\
\hline
\end{tabular}

and load conditions. It can be seen that the transient settling time is around $2.0 \mathrm{~ms}$ for all cases of operations, even in the worst-case operating condition: $v_{\mathrm{i}}=20 \mathrm{~V}$ and step output current change of $0.2 \mathrm{~A}$ to $2.0 \mathrm{~A}$, which has a voltage ripple swing of around $2.4 \mathrm{~V}$ (see Fig. 3(c)). Recalling that in a previous work which uses the same converter specification [12], it is shown that the PWM based SM voltage controller achieves better robustness in wide operating conditions than the peak current mode controller. However, its responses $(4.4 \mathrm{~ms})$ are much slower than the peak current mode controller's response $(2.0 \mathrm{~ms})$ at the nominal condition. Hence, a comparison of the experimental results with those obtained in [12] demonstrates the strength of the proposed PWM based SM current controller in terms of robustness and fast response.

\section{CONCLUSION}

A fixed-frequency pulse-width-modulation based sliding mode current controller for boost converter is presented. The methods of modeling the system and the translation of the sliding mode control equations for the PWM implementation are illustrated. The resulted PWM based sliding mode current controller can be easily realized with simple analog circuitries. It can be concluded from the experimental results that the derived controller/converter system inherits the excellent ro- 


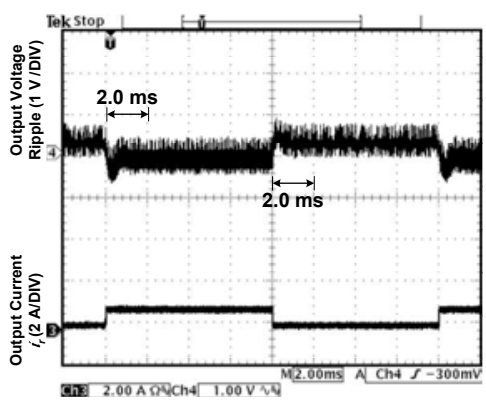

(a) $v_{\mathrm{i}}=20 \mathrm{~V}(0.2 / 1.0 \mathrm{~A})$

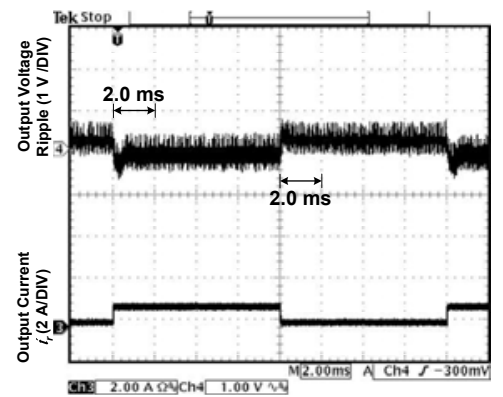

(d) $v_{\mathrm{i}}=24 \mathrm{~V}(0.2 / 1.0 \mathrm{~A})$

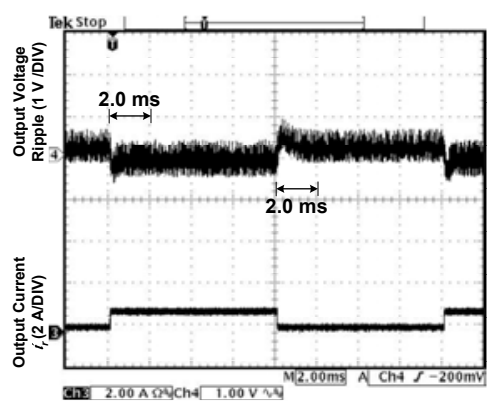

(g) $v_{\mathrm{i}}=28 \mathrm{~V}(0.2 / 1.0 \mathrm{~A})$

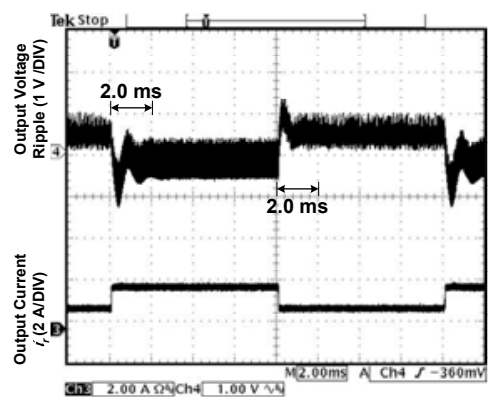

(b) $v_{\mathrm{i}}=20 \mathrm{~V}(1.0 / 2.0 \mathrm{~A})$

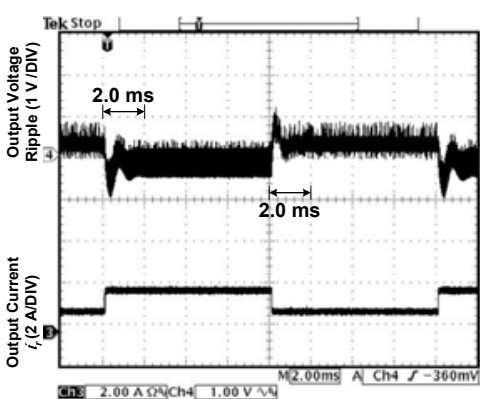

(e) $v_{\mathrm{i}}=24 \mathrm{~V}(1.0 / 2.0 \mathrm{~A})$

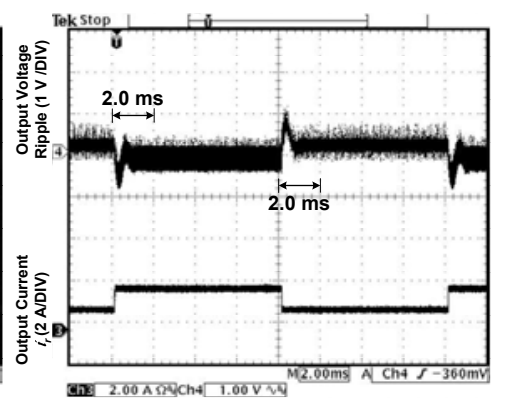

(h) $v_{\mathrm{i}}=28 \mathrm{~V}(1.0 / 2.0 \mathrm{~A})$

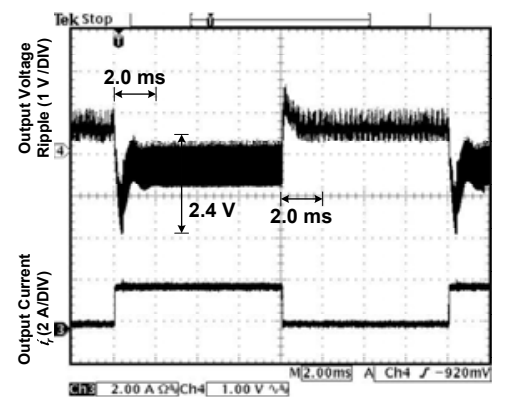

(c) $v_{\mathrm{i}}=20 \mathrm{~V}(0.2 / 2.0 \mathrm{~A})$

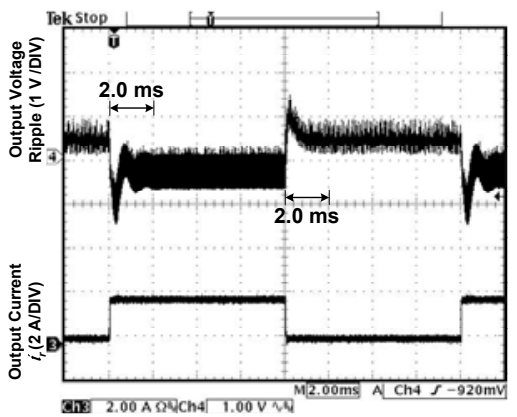

(f) $v_{\mathrm{i}}=24 \mathrm{~V}(0.2 / 2.0 \mathrm{~A})$

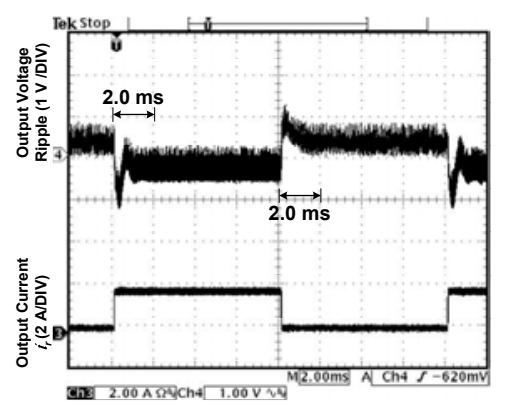

(i) $v_{\mathrm{i}}=28 \mathrm{~V}(0.2 / 2.0 \mathrm{~A})$

Fig. 3. Experimental waveforms of the boost converter with the PWM based SM current controller.

bustness feature of the sliding mode voltage controller, as well as the fast response capability of a current mode controller. However, it has the disadvantages of requiring two current sensors and being a more complex circuit.

\section{REFERENCES}

[1] F.A. Himmelstoss, J.W. Kolar and F.C. Zach, "Analysis of a Smithpredictor-based-control concept eliminating the right-half plane zero of continuous mode boost and buck-boost DC/DC converters," in Proceedings, International Conference on Industrial Electronics, Control and Instrumentation IECON, pp. 423-428, Nov. 1991.

[2] R. Ridley, "Current mode or voltage mode?," in Switching Power Magazine, pp. 4-9, Oct 2000.

[3] R. Mammano, "Switching power supply topology: voltage mode vs. current mode," in Unitrode Design Note, Jun 1994.

[4] R. Venkataramanan, A. Sabanoivc, and S. Ćuk, "Sliding mode control of DC-to-DC converters," in Proceedings, IEEE Conference on Industrial Electronics, Control and Instrumentations (IECON), pp. 251-258, 1985.

[5] V. Utkin, J. Guldner, and J.X. Shi, Sliding Mode Control in Electromechanical Systems. London, U.K.: Taylor and Francis, 1999.

[6] P. Mattavelli, L. Rossetto, G. Spiazzi, and P. Tenti, "General-purpose sliding-mode controller for dc/dc converter applications," in IEEE Power Electronics Specialists Conference Record (PESC), pp. 609-615, June 1993.
[7] V.M. Nguyen and C.Q. Lee, "Indirect implementations of sliding-mode control law in buck-type converters," in Proceedings, IEEE Applied Power Electronics Conference and Exposition (APEC), vol. 1, pp. 111115, March 1996.

[8] J. Mahdavi, A. Emadi, and H.A. Toliyat, "Application of state space averaging method to sliding mode control of PWM DC/DC converters," in Proceedings, IEEE Conference on Industry Applications (IAS), vol. 2, pp. 820-827, Oct. 1997.

[9] C.C. Wu and C.M. Young, "A new PWM control strategy for the buck converter," in Proceedings, IEEE Conference on Industrial Electronics Society (IECON), pp. 157-162, 1999.

[10] S.K. Mazumder and S.L. Kamisetty, "Experimental validation of a novel multiphase nonlinear VRM controller", in IEEE Power Electronics Specialists Conference Record (PESC), pp. 2114-2120, June 2004.

[11] S.C. Tan, Y.M. Lai, C.K. Tse, and M.K.H. Cheung, "A fixed-frequency pulsewidth-modulation based quasi-sliding mode controller for buck converters", IEEE Transactions on Power Electronics, vol. 20, no. 6, pp. 1379-1392, Nov. 2005.

[12] S.C. Tan, Y.M. Lai, and Chi K. Tse, "A unified approach to the design of PWM based sliding mode voltage controller for basic DC-DC converters in continuous conduction mode", IEEE Transactions on Circuits and Systems I, to appear.

[13] H. Sira-Ramirez, "A geometric approach to pulse-width modulated control in nolinear dynamical systems," IEEE Transactions on Automatic Control, vol. 34 no. 3, pp. 184-187, Feb. 1989.

[14] L. Martinez, A. Poveda, J. Majo, L. Garcia-de-Vicuna, F. Guinjoan, J.C. Marpinard, and M. Valentin, "Lie algebras modelling of bidirectional switching converters," in Proceedings, European Conference on Circuit 
Theory and Design (ECCTD), vol. 2, pp. 1425-1429, Sep. 1993.

[15] E. Fossas, L. Martínez and J. Ordinas, " Sliding mode control reduces audiosusceptibility and load perturbation in the Cuk converter," IEEE Transactions on Circuits and Systems Part I: Fundamental Theory and Applications, , vol. 39 no. 10, pp. 847-849, Oct 1992.

[16] L. Martinez-Salamero, J. Calvente, R. Giral, A. Poveda, and E. Fossas, "Analysis of a bidirectional coupled-inductor Ćuk converter operating in sliding mode," IEEE Transactions on Circuits and Systems Part I Fundamental Theory and Applications, vol. 45, no. 4, pp. 355-363, April 1998.

[17] J. Ackermann and V. Utkin, "Sliding mode control design based on Ackermann's formula,' IEEE Transactions on Automatic Control, vol. 43 no. 2, pp. 234-237, Feb. 1998. 